INPLASY

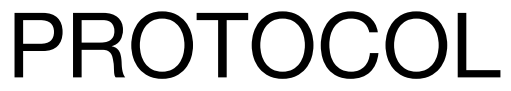

To cite: Liu et al. The efficiency and safety of alendronate versus teriparatide for treatment glucocorticoidinduced osteoporosis: A metaanalysis and systematic review of randomized controlled trials. Inplasy protocol 202090025. doi:

10.37766/inplasy2020.9.0025

Received: 04 September 2020

Published: 06 September 2020

Corresponding author:

Fei Yin

yinfei999@jlu.edu.cn

Author Affiliation:

Department of Spine Surgery, China-Japan Union Hospital of Jilin University, Changchun, China

Support: None.

Review Stage at time of this submission: Data analysis.

Conflicts of interest:

None.

\section{The efficiency and safety of} alendronate versus teriparatide for treatment glucocorticoid-induced osteoporosis: A meta-analysis and systematic review of randomized controlled trials

\author{
Liu, Z1; Zhang, M²; Shen, Z3; Ke, J4.
}

Review question / Objective: Glucocorticoid-induced osteoporosis (GIOP) is the most common secondary osteoporosis, alendronate (ALE) and teriparatide (TPTD) are widely used in the treatment of GIOP. Through this systematic review and meta-analysis, we evaluated the effectiveness and safety of ALE and TPTD in the treatment of GIOP.

Condition being studied: In patients who receive long-term glucocorticoid treatment, $30-50 \%$ may have fractures, especially the TH, LS and FN. The dose and duration of glucocorticoids have a significant effect on the occurrence of fractures. The daily average dose of glucocorticoids seems to be more predictive of the risk of fracture risk than cumulative doses. Taking $2.5 \mathrm{mg}$ of oral prednisone daily increases the risk of fractures, and when the dose is greater than $7.5 \mathrm{mg}$ (the approximate physical amount of endogenous glucocorticoids), the risk increases 5 times. In addition, the risk of fractures increases significantly with the increasing dose of glucocorticoids and the prolongation of time. Longterm treatment and high-dose of glucocorticoids will cause osteoporotic fractures in $30-50 \%$ of adult patients.

INPLASY registration number: This protocol was registered with the International Platform of Registered Systematic Review and Meta-Analysis Protocols (INPLASY) on 06 September 2020 and was last updated on 06 September 2020 (registration number INPLASY202090025).

\section{INTRODUCTION}

Review question / Objective: Glucocorticoid-induced osteoporosis (GIOP) is the most common secondary osteoporosis, alendronate (ALE) and teriparatide (TPTD) are widely used in the treatment of GIOP. Through this systematic review and meta-analysis, we evaluated the 
effectiveness and safety of ALE and TPTD in the treatment of GIOP.

Condition being studied: In patients who receive long-term glucocorticoid treatment, $30-50 \%$ may have fractures, especially the TH, LS and FN. The dose and duration of glucocorticoids have a significant effect on the occurrence of fractures. The daily average dose of glucocorticoids seems to be more predictive of the risk of fracture risk than cumulative doses. Taking $2.5 \mathrm{mg}$ of oral prednisone daily increases the risk of fractures, and when the dose is greater than $7.5 \mathrm{mg}$ (the approximate physical amount of endogenous glucocorticoids), the risk increases 5 times. In addition, the risk of fractures increases significantly with the increasing dose of glucocorticoids and the prolongation of time. Long-term treatment and high-dose of glucocorticoids will cause osteoporotic fractures in $30-50 \%$ of adult patients.

\section{METHODS}

Search strategy: "Glucocorticoid(s)" or"corticoid(s)"or"corticosteroid(s)"or"met hylprednisolone"or"prednisone"or"prednis olone"or"hydrocortisone"or"triamcinolone" or"dexamethasone"and"osteoporosis"and" alendronate"and" teripatide".

Participant or population: Patients had taken prednisone or its equivalent at a dosage of $\geq 5 \mathrm{mg} /$ day for $\geq 3$ months prior to screening.

Intervention: Subjects took teriparatide.

Comparator: Subjects took alendronate.

Study designs to be included: Randomized controlled trials.

Eligibility criteria: (1)Patients were at least 21 years old; (2) Patients had taken prednisone or its equivalent at a dosage of $\geq 5 \mathrm{mg} /$ day for $\geq 3$ months prior to screening; (3)Patients were required to have a LS or TH BMD T score of $\leq-2.0$ or $\leq-1.0$ plus at least one fragility fracture while taking glucocorticoids; (4)Studies' language was English; (5) Studies were RCTs.

Information sources: We will search Pubmed, Embase and Cochrane Library.

Main outcome(s): We found that compared with ALE, TPTD could reduce the rate of new vertebral fracture (RR=0.13, $95 \% \mathrm{Cl}$ : 0.05-0.29, $P<0.00001)$.TPTD increased LS bone mineral density (BMD)(SMD $=0.53$, 95\% Cl 0.44-0.63, P<0.00001), TH BMD (SMD $=0.17,95 \%$ Cl 0.07-0.27, $\mathrm{P}<0.00001$ ) and FN BMD $(0.17 \%, 95 \%$ Cl $0.05-0.29$, $\mathrm{P}<0.00001)$ compared to ALE. However, there was no significant difference in the incidence of non-vertebral fracture and adverse events between the two groups.

Data management: In this paper, two researchers independently conducted literature search, screening, data extraction and heterogeneity analysis. If there is any objection, we will reach an agreement after discussion, complete the preliminary search according to the established search strategy, and read the abstract and full text to exclude studies that do not meet the inclusion criteria.

Quality assessment / Risk of bias analysis: Two or three review authors will use the Cochrane 'Risk of Bias' tool to independently assess the risk of bias included in the study. We will assess the risk of deviations such as random sequence generation, allocation hiding, blindness of participants and personnel, blinding of outcome assessment, incomplete result data, selective reports, and other sources of bias.

Strategy of data synthesis: The primary outcome of this study was the incidence of vertebral fracture and non-vertebral fracture, while the secondary outcome was the mean percent changes from baseline to $6,12,18$ months in the ALE and TPTD groups in lumbar spine(LS) bone mineral density (BMD), the mean percent changes from baseline to 18 months in the two groups in the BMD of the femoral neck (FN) and total hip (TH), the incidence of 5 major adverse events, and changes in bone 
formation and resorption markers in both groups. In this paper, two researchers independently conducted literature search, screening, data extraction and heterogeneity analysis. If there is any objection, we will reach an agreement after discussion, complete the preliminary search according to the established search strategy, and read the abstract and full text to exclude studies that do not meet the inclusion criteria.

Subgroup analysis: We analyzed the mean percent changes from baseline to $6,12,18$ months in the ALE and TPTD groups in lumbar spine(LS) bone mineral density (BMD).

Sensibility analysis: We will conduct a sensitivity analysis of vertebral and nonvertebral fractures and evaluate the credibility of the results of Meta analysis by excluding studies with a high risk of bias.

Language: English only.

Country(ies) involved: China.

Keywords: Glucocorticoid-induced osteoporosis; Vertebral fracture; Alendronate; Teriparatide; Randomized controlled trial.

Contributions of each author:

Author 1 - Zhiming Liu - participated in drafting manuscripts or critically revised important content.

Author 2 - Min Zhang - make a significant contribution to data acquisition, data analysis and interpretation.

Author 3 - Zhubin Shen - make a significant contribution to data acquisition, data analysis and interpretation.

Author 4 - Junran Ke - make a significant contribution to data acquisition, data analysis and interpretation.

Author 5 - Ding Zhang - make a significant contribution to data acquisition, data analysis and interpretation.

Author 6 - Fei Yin - revised the manuscript and finally approved the version to be released. 